\title{
Efficacy and safety of regorafenib and fruquintinib as third-line treatment for colorectal cancer: a narrative review
}

\author{
Xiaojing Xu\#, Yiyi Yu\#, Mengling Liu, Li Liang, Tianshu Liu^^ \\ Department of Oncology, Zhongshan Hospital affiliated to Fudan University, Shanghai, China \\ Contributions: (I) Conception and design: X Xu, T Liu; (II) Administrative support: T Liu; (III) Provision of study materials or patients: X Xu, Y Yu; (IV) \\ Collection and assembly of data: X Xu, Y Yu, M Liu; (V) Data analysis and interpretation: X Xu, M Liu, T Liu; (VI) Manuscript writing: All authors; \\ (VII) Final approval of manuscript: All authors. \\ "These authors contributed equally to this work. \\ Correspondence to: Prof. Tianshu Liu. Department of Oncology, Zhongshan Hospital affiliated to Fudan University, 180 Fenglin Road, Shanghai \\ 200032, China. Email: liutianshu1969@126.com.
}

Objective: The efficacy and safety of regorafenib and fruquintinib are studied extensively in different populations and trials across the world to determine their potential benefits. Here we review the efficacy and safety of regorafenib and fruquintinib as third-line treatment option for colorectal cancer (CRC).

Background: CRC is the third most prevalent cancer worldwide, but the optimal third-line treatment option is still debatable. Regorafenib is a multikinase inhibitor that inhibits several cell signaling receptors, including vascular endothelial growth factor receptors (-1, -2, and -3) (VEGFRs), platelet-derived growth factor (PDGF), fibroblast growth factor, epidermal growth factor (EGF), angiotensin II, and Rapidly Accelerated Fibrosarcoma kinase pathway. On the other hand, fruquintinib inhibits signaling through the VEGFRs family. Regorafenib and fruquintinib have both received United States Food and Drug Administration (USFDA) approval for treating metastatic CRC (mCRC) in patients previously treated with fluoropyrimidine-, oxaliplatin-, and irinotecan-based chemotherapy, an anti- vascular endothelial growth factor therapy, and Rat sarcoma wild type, an anti- EGF receptor therapy.

Methods: A review of literature was conducted in PubMed and Embase with the keywords "regorafenib" OR "fruquintinib" AND "colorectal cancer" for clinical studies performed in randomized controlled settings and real-world settings.

Conclusions: Regorafenib and fruquintinib are effective and well tolerated options for the third-line treatment of patients with CRC. Both have a similar survival outcome with Regorafenib showing a slightly better toxicity profile.

Keywords: Metastatic colorectal cancer (mCRC); regorafenib; fruquintinib; clinical trials

Submitted Dec 30, 2020. Accepted for publication Aug 26, 2021.

doi: $10.21037 /$ tcr-20-3539

View this article at: https://dx.doi.org/10.21037/tcr-20-3539

\section{Introduction}

Colorectal cancer (CRC) is the third most prevalent cancer $(1,931,590$ cases in 2020) and the second leading cause of cancer-related death $(935,173$ deaths in 2020) worldwide (1). Although it is more common in high- income countries, the rate of its incidence and related mortality is rapidly increasing in many low- and middleincome countries as well (2). The global prevalence of CRC is higher in men than in women $(746,298 v s .614,304)(3)$. Furthermore, men have significantly higher rates of CRC-

\footnotetext{
^ ORCID: 0000-0002-9626-8874.
} 
related mortality than women $(372,639$ vs. 320,294$)$, and this difference is observed across all age groups (4). Of all patients with CRC, metastases are detected in $25 \%$ of patients at the time of diagnosis, whereas $50 \%$ subsequently advance to metastases, accounting for a higher mortality rate (5).

Development and implementation of targeted therapies in the past 15 years have led to increased overall survival (OS) and response rate among patients with metastatic CRC (mCRC) $(6,7)$. The standard first-line and second-line therapies for patients with mCRC consist of two or three chemotherapy drugs combined with a targeted drug $(7,8)$. Targeted drugs used for mCRC treatment are directed against epidermal growth factor receptor (anti-EGFRs) or vascular endothelial growth factor receptors (anti-VEGFRs), both of which inhibit angiogenesis and thus limit tumor growth and metastasis $(9,10)$.

Regorafenib, an oral multikinase inhibitor, has been approved by the United States Food and Drug Administration (USFDA) as third- or later-line therapy for patients who respond poorly to first- and secondline treatments (11). Its approval was based on the results of the CORRECT trial (12). It is also approved by the European Medicines Agency and the China Food and Drug Administration $(13,14)$. Recently, another oral VEGFR inhibitor, fruquintinib, was also approved by the USFDA for third-line treatment of mCRC (15).

Regorafenib and fruquintinib differ in their mechanism of action (16-19). Regorafenib blocks cell signaling pathways involving VEGFR-1, -2, and -3, fibroblast growth factor receptor (FGFR), platelet-derived growth factor (PDGF)- $\alpha$ and $-\beta$, epidermal growth factor (EGF), angiotensin 2 , and RAF (20). On the other hand, fruquintinib which represents a new generation of small-molecule VEGFR inhibitors selectivity targets VEGFR-1, -2 , and -3 only (17).

Although randomized clinical trials (RCTs) have shown that both regorafenib and fruquintinib significantly improve survival outcomes in patients with mCRC when compared with placebo, studies are underway to illustrate the headon comparison between the two. One such real-world study conducted at the Peking University comprises a cohort of regorafenib versus fruquintinib in $\mathrm{mCRC}$ patients who have progressed after at least one second-line chemotherapeutic intervention (NCT04431791). In the current review, the safety and efficacy of regorafenib and fruquintinib as thirdline treatment for patients with mCRC using evidence from RCTs and observational studies will be summarized. We present the following article in accordance with the
Narrative Review reporting checklist (available at https:// tcr.amegroups.com/article/view/10.21037/tcr-20-3539/rc).

\section{Methods}

Literature search was done on PubMed for articles published in English focusing on relevant articles. The keywords "regorafenib" OR "fruquintinib" AND "colorectal cancer" for clinical studies performed in randomized controlled settings and real-world settings were used.

\section{Targeting angiogenesis in CRC}

Angiogenesis, that is, the formation of new blood vessels, is imperative for tumor growth and metastasis $(21,22)$. It is a complex process involving the interplay of multiple proand anti-angiogenic factors and cell signaling pathways. The expression level of these factors in cells is controlled by hypoxia response elements on DNA molecule, which are activated by hypoxia-inducible factor (transcription factor) under deprivation of adequate oxygen supply conditions (23). The classical factors involved in angiogenesis are VEGF family, PDGF, EGF, hepatocyte growth factor, insulin-like growth factors, angiopoietins, fibroblast growth factors, tumor necrosis factor, transforming growth factors, and interleukins $(22,23)$. These angiogenic factors act via tyrosine kinase receptors and downstream signaling proteins through the RAF kinase pathway or PI3K/PTEN/Akt pathway (23). EGFR signaling plays a pivotal role in tumor growth and progression in CRC (24). EGFR, when activated (via ligand binding), triggers PI3K/PTEN/Akt and Ras-Raf-MEK-MAPK pathways to promote tumor cell proliferation, angiogenesis, and metastasis (24).

Clinical studies have shown that anti-angiogenic drugs such as anti-VEGFR and anti-EGFR can improve the survival of patients with mCRC $(10,25)$. According to a systematic review and meta-analysis of $12 \mathrm{RCTs}$ comprising 7,108 patients, cetuximab, an EGFR inhibitor, can significantly prolong progression-free survival (PFS) and OS in patients with mCRC in combination with chemotherapeutic agents versus chemotherapy alone (26). In addition, REVERCE was a phase 2 randomized study on the sequential treatment of regorafenib followed by cetuximab compared with cetuximab followed by regorafenib in patients with mCRC (UMIN000011294). The results from this study showed that patients in the regorafenib followed by cetuximab arm 
had better PFS [PFS: 5.2 vs. 1.8 months; hazard ratio (HR): 0.29] and OS (17.4 vs. 11.6 months; HR: 0.61, $\mathrm{P}=0.03$ ) than those who received cetuximab before regorafenib (27). However, tumors develop resistance toward bevacizumab, which is an anti-VEGFR monoclonal antibody activating alternative angiogenic pathways (28-31). Hayashi et al. [2014] evaluated serum concentrations of biomarkers in patients with $\mathrm{mCRC}$ before and after treatment with folinic acid, fluorouracil, and irinotecan (FOLFIRI regimen) and bevacizumab (32). They reported that after treatment, VEGF-A levels decreased significantly $(\mathrm{P}<0.0001)$; however, a concurrent and significant increase in PDGF concentration was also observed $(\mathrm{P}<0.0001)$. Interestingly, the treatment led to significantly higher levels of VEGF-C after 1 month and VEGF-D after 2 and 6 months of therapy compared with baseline levels (the period following treatment) (32).

Thus, it is suggested that drugs that can simultaneously block multiple signaling pathways should be used for effective inhibition of angiogenesis (33-35). VEGFR and EGFR signaling pathways are interlinked, and hence, two approaches are used to inhibit both pathways: (I) a horizontal approach consisting of an anti-angiogenic drug that can block signaling through VEGFR as well as EGFR simultaneously; (II) a vertical approach that utilizes two different drugs to block both VEGFR- and EGFRmediated signaling. As VEGFR and EGFR pathways are interdependent, the synergistic inhibition might limit the possibility of resistance to a targeted therapy that is observed with the usage of a single targeted drug $(33,35,36)$.

\section{Regorafenib and fruquintinib as anti-angiogenic agents in $\mathrm{mCRC}$}

Regorafenib inhibits signaling through VEGFR-1, -2, and -3, PDGFR $\beta$, c-KIT, RET, FGFR1, and TIE2, and thereby, limits tumor progression. In addition, it inhibits the RAF/ MEK/MAPK cascade, which is involved in downstream signaling of these receptors and EGFR (Figure 1). Cell signaling through these receptors and kinases is required for tumor growth and angiogenesis. Hence, by inhibiting signaling through these pathways, regorafenib can effectively limit tumor progression in CRC $(16,20)$. On the other hand, fruquintinib, a potent, small-molecule VEGFR inhibitor used for mCRC treatment, targets VEGFR-1, -2 , and -3 only. It inhibits VEGF-induced VEGFR2 phosphorylation, which is required for downstream signaling, leading to angiogenesis and tumor growth (Figure 1) $(15,17)$. As regorafenib targets multiple pathways, it can inhibit angiogenesis more profoundly than fruquintinib and also limit the possibility of development of resistance in tumor cells toward targeted therapy $(33,37)$. Based on this, regorafenib could clinically be a more effective anti-angiogenic agent in $\mathrm{mCRC}$ compared with fruquintinib.

\section{Efficacy of regorafenib and fruquintinib in third-line therapy of $m C R C$}

\section{Evidence from randomized controlled trials}

Two large randomized phase 3 trials, CORRECT (NCT01103323) and CONCUR (NCT01584830), have demonstrated that regorafenib effectively prolongs the median OS and PFS in patients with mCRC compared with placebo $(12,38)$. CORRECT was an international multicenter, randomized, phase 3 trial that enrolled patients with mCRC from 114 centers across 16 countries (12). Patients were randomized to receive either regorafenib $(n=505)$ or placebo $(n=255)$, and it was seen that OS and PFS were significantly extended in regorafenib-treated patients than in those receiving placebo (OS: 6.4 vs. 5.0 months; HR: 0.77, 95\% CI: 0.64-0.94, P=0.0052; PFS: 0.49, 95\% CI: $0.42-0.58, \mathrm{P}<0.0001)(12)$. As 111 patients in the CORRECT trial were of Asian ethnicity, the results confirmed the efficacy of regorafenib in Asian patients with mCRC. In another trial, CONCUR, 204 Chinese patients from 25 hospitals were randomized to receive oral regorafenib (160 mg, daily) or placebo on days 1 to 21 of each 28-day cycle. Patients included in the trial had received at least two lines of treatment for $\mathrm{mCRC}$. The regorafenibtreated group had significantly longer OS compared with the placebo group (8.8 vs. 6.3 months; HR: 0.55, 95\% CI: $0.40-0.77, \mathrm{P}=0.00016)$. Similarly, PFS was prolonged in the regorafenib group compared with the placebo group (3.2 vs. 1.7 months; HR: 0.31, 95\% CI: 0.22-0.44, $\mathrm{P}<0.0001)$.

Efficacy data for fruquintinib are mostly obtained from a placebo-controlled phase 3 trial, FRESCO (NCT02314819), which randomized 416 Chinese patients with $\mathrm{mCRC}$ to receive either fruquintinib $(\mathrm{n}=278$; dosage $5 \mathrm{mg} /$ day) or placebo ( $\mathrm{n}=138$; dosage $5 \mathrm{mg} /$ day) for 1 to 21 days in a course of 28-day cycle with best supportive care. Fruquintinib was efficacious in significantly prolonging $\mathrm{OS}$ in comparison with placebo (9.3 vs. 6.6 months; HR: 0.65 , 95\% CI: $0.51-0.83, \mathrm{P}<0.001)$ and PFS (3.7 vs. 1.8 months; HR: 0.26, 95\% CI: 0.21-0.34, $\mathrm{P}<0.001)$. Interestingly, OS did not differ between the treatment arms among patients aged $\geq 65$ years (HR: 0.95 , 


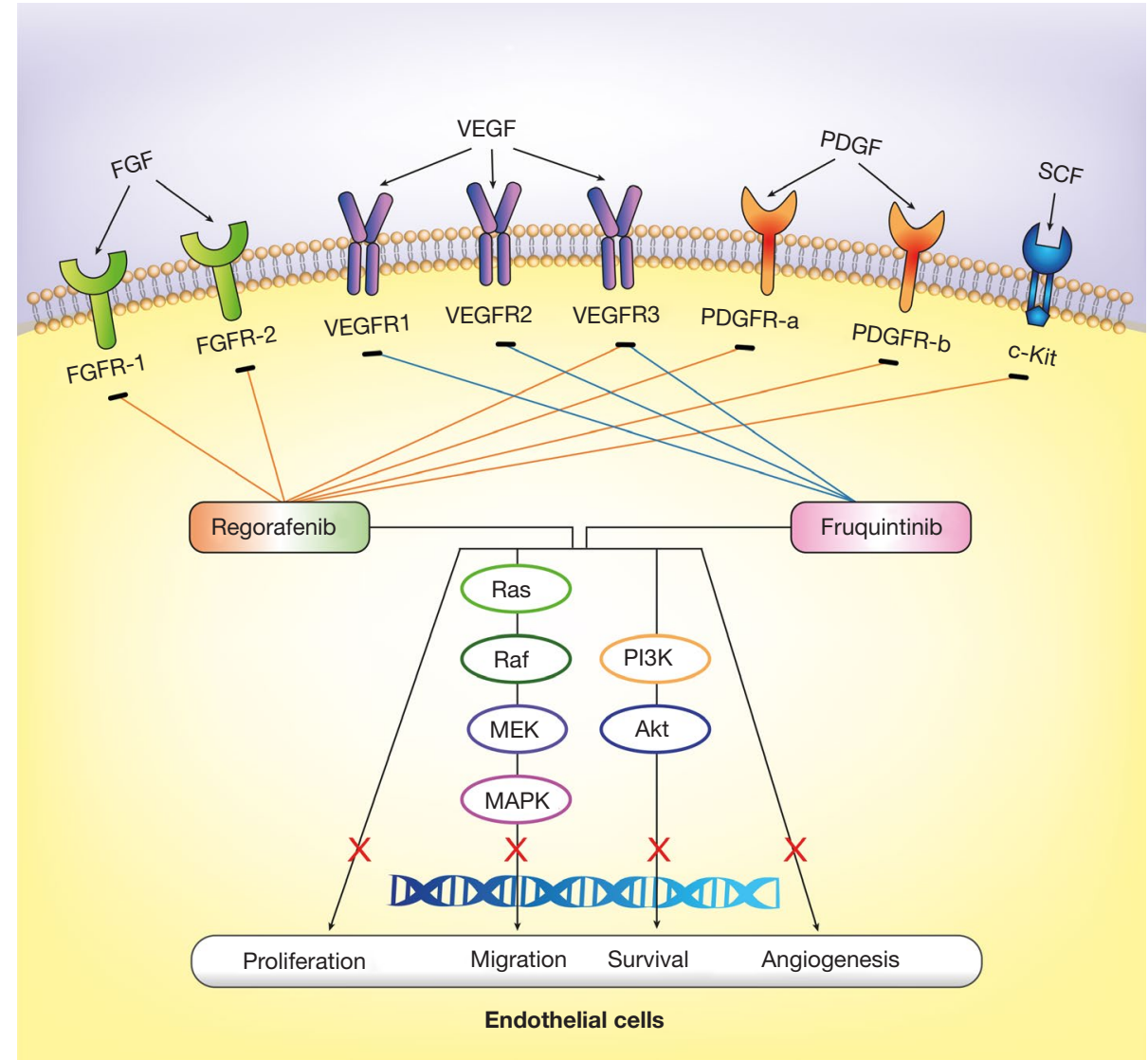

Figure 1 Mechanism of action for regorafenib and fruquintinib.

95\% CI: $0.55-1.63)$ (39). This finding implies that the treatment effect in terms of OS was better in FRESCO compared with CONCUR primarily because of the inclusion of a higher proportion of patients aged $\geq 65$ years in the regorafenib group in the former study (12). This possibility was further confirmed by the subgroup analysis that indicated better survival in patients aged $<65$ years than in those aged $\geq 65$ years in CORRECT (OS: 0.86 vs. 0.72; PFS: 0.65 vs. 0.42 ) and FRESCO trials (OS: $0.95 v s$. 0.56 ; PFS: 0.33 vs. 0.26). In addition to the aforementioned factor of age, it is worth noting that the FRESCO trial consisted of more men in its placebo group than the fruquintinib group, thereby skewing the analysis in favor of fruquintinib, as shown by the subgroup analysis wherein similar OS was observed in the fruquintinib and placebo arms among women (HR: 0.85, 95\% CI: 0.57-1.29). Most importantly, the FRESCO trial included fewer patients than the CORRECT trial (416 vs. 760, respectively). Moreover, the CORRECT trial was an international trial that included patients from various ethnicities (16 countries), whereas the FRESCO trial consisted of only Chinese patients with mCRC, thus limiting its global generalizability $(38,39)$. On the other hand, the CONCUR study included a lower proportion of patients with no history of targeted therapy (41\% vs. $60.1 \%)$ and a higher proportion of patients aged $>65$ years $(30 \%$ vs. $18 \%)$ in the treatment arm than in the placebo arm when compared with FRESCO. Although both CONCUR and FRESCO showed a survival benefit with both the drugs, a greater reduction in the risk of death was observed in CONCUR with regorafenib than in FRESCO with fruquintinib (45\% vs. 35\%).

Furthermore, in the randomized, phase $2 \mathrm{~b}$ study by $\mathrm{Xu}$ et al., 47 Chinese patients with mCRC received fruquintinib, whereas 24 were given a placebo, in addition to best supportive care. The patients enrolled had received $\geq 2$ lines of prior therapies for mCRC. OS was similar among patients in the fruquintinib arm and those in the placebo arm (HR: 0.71; 95\% CI: 0.38-1.34). However, PFS was significantly better in the fruquintinib arm (HR: 0.30, 95\% CI: 0.15-0.59, $\mathrm{P}<0.001)(40)$. 
Evidence from real-world/retrospective studies

As RCTs usually evaluate the efficacy and safety in a controlled setting, data on these parameters from reallife prescribing conditions may differ. Thus, evidence from real-world cohort studies is of utmost importance to understand the efficacy, safety, and potential predictors of outcome in patients treated with regorafenib. REgorafeniB in mEtastatic Colorectal cancer: a French Compassionate progrAm (REBECCA) is a cohort study that evaluated the efficacy and safety of regorafenib in French patients with mCRC ( $n=654)$ who were previously treated with or were not considered candidates for standard therapies (41). Of the total population, $35 \%$ had received at least three prior lines of therapy for mCRC and $10.6 \%$ of patients had Eastern Cooperative Oncology Group performance status (ECOG PS) scores $\geq 2$. In the low-risk group $(n=213)$, regorafenib led to a median OS of 9.2 months with a 6-month OS rate of $67 \%$, highlighting the importance of identifying subgroups of patients with mCRC who can benefit most from regorafenib (41). Furthermore, the researchers performed a post hoc analysis in the subgroup of patients who had baseline characteristics similar to that of the CORRECT study population. The median OS in this subgroup ( $\mathrm{n}=440)$ was 6.3 months, whereas in the low-risk population, it was 8.7 months (6-month OS rate of $64 \%$ ).

Kopeckova et al. analyzed data on 148 regorafenibtreated patients with mCRC from the National Clinical Registry of the Czech Republic and reported median OS and PFS of 9.3 and 3.5 months, respectively. Almost all patients (97.8\%) included in the analysis had an ECOG PS score of 0-1 (42). Efficacy data from trials of regorafenib and fruquintinib are summarized in Table 1. Safety of regorafenib and fruquintinib for third-line therapy of mCRC.

Signaling through tyrosine kinase receptor is required in both tumor and normal cells, and thus, the use of tyrosine kinase inhibitors (TKIs) is associated with undesired side effects (23). Hence, to determine the safety of a drug, an important consideration would be whether the resultant adverse events (AEs) were manageable or not. None of the studies evaluating the efficacy and safety of regorafenib or fruquintinib have reported the frequency of drug-related manageable AEs. However, serious AEs have been reported by five studies (CORRECT, CONCUR, RECORA, FRESCO, and a study by $\mathrm{Xu}$ et al.). Of these, CONCUR, FRESCO, and the study by $\mathrm{Xu}$ et al. were conducted in patients of Asian ethnicity. Tables 2,3 present the safety data on regorafenib and fruquintinib. Data from the phase
3 CONCUR trial have strengthened the safety data on regorafenib. The trial randomly assigned 204 patients to receive either regorafenib or placebo (38). The proportion of patients experiencing drug-related AEs in the regorafenib and placebo arms was $97 \%$ and $46 \%$, respectively. Handfoot skin reaction (HFSR) was the most frequent $\mathrm{AE}$ (73\%). The common grade 3 or higher regorafenib-related AEs were HFSR (16\%) followed by hypertension (HTN; 11\%), hyperbilirubinemia (7\%), and hypophosphatemia (7\%). Serious drug-related AEs occurred in 9\% of regorafenibtreated patients and $4 \%$ of patients receiving placebo.

On the other hand, in the FRESCO trial consisting of Chinese patients, $99 \%$ in the fruquintinib arm and $88 \%$ in the placebo arm had treatment-related AEs. In addition, $16 \%$ of the patients receiving fruquintinib and $6 \%$ of those treated with placebo experienced serious AEs, which are higher in comparison with those observed in the CONCUR trial (39). Grade $\geq 3$ treatment-emergent AEs (TEAEs) occurred in $61.2 \%$ of patients receiving fruquintinib and $19.7 \%$ of those receiving a placebo. Approximately $15.5 \%$ and $5.8 \%$ of patients reported serious AEs in the fruquintinib and placebo groups, respectively; $14.4 \%$ of fruquintinib-treated patients experienced serious AEs requiring hospitalization compared with $5.1 \%$ of those treated with placebo. Moreover, TEAEs proved fatal in $3.2 \%$ of fruquintinib-treated patients and $1.5 \%$ of those receiving placebo. The most common $\mathrm{AE}$ reported was HTN (30\%) followed by fatigue (4\%), HFSR (15\%), and diarrhea (2\%).

Importantly, the proportion of patients aged $<65$ years in the drug arm was $82 \%$ in FRESCO compared with $70 \%$ in CONCUR $(38,39)$. The CONCUR trial included more patients with $>3$-line systemic therapy (54\% vs. 31.7\%) or metastatic disease treatment ( $38 \%$ vs. $20.5 \%)$. These patients had poor prognosis, which could have negatively affected the safety profile of the drug.

Furthermore, the incidence of serious AEs reported with fruquintinib in CONCUR was lower than that reported in a recent randomized, phase 2 trial in Chinese patients with mCRC who received the best supportive care in addition to the drug or placebo (40). This study reported serious AEs in $25.5 \%$ of patients in the fruquintinib group and $20.8 \%$ of those in the placebo group. Three patients in the fruquintinib group and two in the placebo group died due to AEs.

Notably, post-market surveillance in Japanese patients with mCRC demonstrated that the incidence and grade of AEs with regorafenib peaked at the initial 2 months and 
Table 1 Efficacy of regorafenib and fruquintinib

\begin{tabular}{|c|c|c|c|c|c|c|}
\hline Study name & Study location & $\begin{array}{c}\text { No. of } \\
\text { patients }\end{array}$ & $\begin{array}{c}\text { Median OS, } \\
\text { months }(95 \% \mathrm{Cl})\end{array}$ & OS HR (95\% Cl) & $\begin{array}{c}\text { Median PFS, } \\
\text { months }(95 \% \mathrm{Cl})\end{array}$ & PFS HR (95\% Cl) \\
\hline \multicolumn{7}{|l|}{ Regorafenib } \\
\hline CONCUR (38) & Asia & 136 & $8.8(7.3,9.8)$ & $0.55(0.40,0.77)$ & $3.2(2.0,3.7)$ & $0.31(0.22,0.44)$ \\
\hline Japan PMS (44) & Japan & 1227 & $7(6.3,7.8)$ & - & - & - \\
\hline CORRELATE (45) & Global & 1037 & $7.6(7.1,8.2)$ & - & $2.8(2.6,2.8)$ & - \\
\hline RECORA (46) & Germany & 481 & $5.8(5.3,6.6)$ & - & $3.1(2.8,3.3)$ & - \\
\hline Fruquintinib & & & & - & & \\
\hline FRESCO phase 2 trial (39) & China & 278 & 9.30 & $0.65(0.51,0.83)$ & 3.71 & $0.26(0.21,0.34)$ \\
\hline Phase 2 trial (40) & China & 47 & 7.72 & & 4.73 & - \\
\hline
\end{tabular}

$\mathrm{Cl}$, confidence interval; HR, hazard ratio; OS, overall survival; PFS, progression free survival; PMS, post-marketing surveillance.

stabilized afterward (44). This finding is supported by the results of the CORRECT study over the time course of regorafenib-associated AEs (43).

\section{Regorafenib: safety from studies with a majority of Caucasian patients}

In the CORRECT trial, the incidence of drug-related AEs leading to a dose reduction $38 \%$ in the regorafenib arm and $3 \%$ in the placebo arm) and grade $\geq 3$ AEs (54\% in the regorafenib arm and $14 \%$ in the placebo arm) compared with placebo was lower than that reported with fruquintinib in the FRESCO trial $(12,39)$. It was observed that among regorafenib-treated patients, the incidence of AEs peaked early during treatment and gradually decreased with each cycle of treatment (43). The incidence of HFSR, fatigue, diarrhea, HTN, and rash reduced from $32 \%, 45 \%, 23 \%$, $21 \%$, and $24 \%$ after the first cycle of treatment to $25 \%$, $22 \%, 18 \%, 2 \%$, and $1 \%$ after six cycles of treatment, respectively. The proportion of patients with serious AEs was similar in the regorafenib and placebo groups (44\% and $40 \%$, respectively). The common AEs observed are listed in Table 3.

Furthermore, the findings from CORRELATE, a prospective, observational study, showed that $80 \%$ of patients treated with regorafenib had drug-related AEs and $36 \%$ suffered from grade $\geq 3$ AEs. The incidence of AEs was relatively low compared with other studies, which the authors attributed to the fact that almost half of the patients received less than the recommended daily dose $(160 \mathrm{mg} /$ day) of regorafenib (45). The real-world analysis of the CORRELATE trial in the Taiwanese cohort depicted a consistent safety report, as observed among Asian patients in trials such as the CORRECT and the CONCUR studies, suggesting HFSR occurring in $33.59 \%$ of patients (51). The common AEs observed with regorafenib use were consistent with other studies (Table 2). Further, the RECORA study determined the real-world efficacy and safety of regorafenib in German patients with mCRC (46). The study reported the occurrence of regorafenib-related grade $\geq 3 \mathrm{AEs}$ and serious $\mathrm{AEs}$ in $19 \%$ and $9 \%$ of patients, respectively (Table 2).

Calcagno et al. retrospectively investigated the effectiveness and safety of regorafenib in French patients with mCRC. The spectrum of regorafenib-induced AEs was consistent with other studies $(12,38,43,47)$. A median treatment duration of 2.5 months (range, $0.13-11.4$ months) led to treatment-related AEs in $86 \%$ of patients, with the incidence of fatigue, diarrhea, and HFSR in $35 \%, 20 \%$, and 
Table 2 Proportion of patients experiencing adverse events with regorafenib and fruquintinib

\begin{tabular}{|c|c|c|c|c|c|c|c|}
\hline Study name & $\begin{array}{l}\text { Median/average } \\
\text { daily dose (mg) }\end{array}$ & $\begin{array}{l}\text { Any treatment- } \\
\text { related } A E\end{array}$ & $\begin{array}{l}\text { AEs leading to } \\
\text { dose reduction }\end{array}$ & $\begin{array}{c}\text { AEs leading } \\
\text { to treatment } \\
\text { discontinuation }\end{array}$ & Grade $\geq 3$ AEs & $\begin{array}{c}\text { Any serious } \\
\text { AEs }\end{array}$ & $\begin{array}{c}\text { Death during } \\
\text { treatment }\end{array}$ \\
\hline CORRECT $(12,43)$ & $160 / 147.1$ & $93 ; 61$ & $38 ; 3$ & 0 & $54 ; 14$ & $44 ; 40$ & $2 ; 1$ \\
\hline CORRECT (sixth cycle) ${ }^{\dagger}$ & & $100 ; 97^{\S}$ & $67 ; 23$ & - & $78 ; 49$ & - & 0 \\
\hline REBECCA (41) & $N A / 142.2$ & 80 & - & - & 44 & - & 0 \\
\hline $\begin{array}{l}\text { Kopeckova et al., } \\
2017 \text { (42) }\end{array}$ & NA & 11.5 & - & - & 3 & - & 0 \\
\hline Japan PMS (44) & $N A / 131$ & 89 & - & - & - & - & 0 \\
\hline Calcagno et al., 2016 (47) & NA & 86 & & & 34 & & \\
\hline CONSIGN (48) & $160 / 146$ & 91 & 46 & 9 & 57 & - & 0 \\
\hline Lam et al., $2016^{\S}(49)$ & NA & 100 & 78 & - & 60 & - & 0 \\
\hline RAVELLO (50) & NA & - & $64 ; 30$ & $<1 ;<1$ & NA & - & 0 \\
\hline \multicolumn{8}{|l|}{ Fruquintinib (I or I; C) ${ }^{\star}$} \\
\hline FRESCO phase 3 trial (39) & 5 & $\begin{array}{l}96 ; 71 \\
99 ; 88^{\S}\end{array}$ & $24 ; 4$ & $15 ; 6$ & $46 ; 7$ & $\begin{array}{l}16 ; 6 \\
6 ; 2^{\ddagger}\end{array}$ & $3 ; 2$ \\
\hline Xu et al., 2017 (40) & 5 & $93.6 ; 58.3 \%$ & $28 ; 0$ & - & - & $26 ; 21$ & $3 ; 2$ \\
\hline
\end{tabular}

${ }^{*}$, for studies reporting safety data on drug and placebo separately, data from both groups are provided; ${ }^{\dagger}$, AEs following the sixth cycle of treatment; ${ }^{\ddagger}$, treatment-related serious AEs; ${ }^{\S}$, treatment-emergent AEs. AE, adverse event; I, intervention; C, placebo.

$20 \%$ of patients, respectively (Tables 2,3) (47).

The recent single-arm, open-label, phase $3 \mathrm{~b}$ CONSIGN study (NCT01538680) evaluated the safety of regorafenib in patients with mCRC (48). The study enrolled 2872 patients (83\% Caucasian) with mCRC who progressed after standard treatment. The ECOG PS scores of all patients were $\leq 1$. After a median regorafenib treatment duration of 3 months, TEAEs were observed in $91 \%$ of patients. AEs led to dose reduction and treatment discontinuation in $46 \%$ and $9 \%$ of patients, respectively. The most frequent AEs were fatigue (46\%), HFSR (42\%), and HTN (30\%). However, there was no drug-related serious AEs or death reported in the study (48).

Interestingly, when the initial dose of regorafenib was adjusted in these studies, the incidence of grade $\geq 3 \mathrm{AEs}$ and serious AEs reduced, whereas the tolerance of patients to regorafenib increased. An adjustment of the regorafenib dose in CORRECT (76\%) (12), CONCUR (75\%) (38), RECORA
(43\%) (46), CONSIGN (87\%) (48), REBECCA (50\%) (41), and the phase 2 study by Kudo et al. (55\%) (52) led to grade $\geq 3$ $\mathrm{AE}$ or $\mathrm{SAE}$ incidence rates of $54 \%, 54 \%, 19 \%, 63 \%, 43.7 \%$, and $55 \%$, respectively. Thus, regorafenib-related AEs can be limited by titrating the dose based on a patient's response.

Considering that TKIs act on cancer as well normal cells (23), their use is associated with side effects such as HTN, renal vascular injury, and heart failure (23). The most frequent cardiovascular adverse effect observed with TKIs is HTN, which is related to a TKI-induced reduction in Nitric oxide synthesis. Importantly, the most common complication caused by fruquintinib was HTN, which could be difficult to manage, whereas regorafenib usage most commonly led to HFSR, which can be easily managed.

\section{Important considerations}

The ethnicity of patients is an important factor that 
Table 3 Treatment related adverse events with regorafenib and fruquintinib

\begin{tabular}{|c|c|c|c|c|c|c|c|c|c|c|}
\hline Study name & HTN & Fatigue & HFSR & Diarrhea & Anorexia & $\begin{array}{c}\text { Oral } \\
\text { mucositis }\end{array}$ & Nausea & $\begin{array}{c}\text { Decreased } \\
\text { platelet count }\end{array}$ & Thrombocytopenia & Anemia \\
\hline \multicolumn{11}{|l|}{ Regorafenib (I or I; C) } \\
\hline $\begin{array}{l}\text { CORRECT (sixth } \\
\text { cycle }^{\dagger} \text { ) }\end{array}$ & 2 & 22 & 25 & 18 & - & - & - & - & - & - \\
\hline REBECCA (41) & 11 & 41 & 29 & 19 & 15 & 11 & - & - & 3 & - \\
\hline $\begin{array}{l}\text { Kopeckova et al., } \\
2017 \text { (42) }\end{array}$ & - & 2 & 5 & - & - & - & - & - & - & - \\
\hline Japan PMS (44) & 27 & 14 & 56 & - & - & - & - & 17 & - & - \\
\hline CONSIGN (48) & 30 & 46 & 42 & 25 & 24 & 25 & 11 & - & - & 3 \\
\hline Lam et al., 2016§ (49) & 44 & 42 & 78 & 18 & 31 & 4 & - & 20 & 53 & 47 \\
\hline RAVELLO (50) & $55 ; 20$ & $55 ; 60$ & $45 ; 30$ & $18 ; 30$ & $0 ; 10$ & $18 ; 0$ & $0 ; 30$ & - & - & $0 ; 10$ \\
\hline \multicolumn{11}{|l|}{ Fruquintinib $\left(\mathrm{I}\right.$ or I; C) ${ }^{\star}$} \\
\hline $\begin{array}{l}\text { FRESCO phase } 3 \\
\text { trial (39) }\end{array}$ & $55 ; 15$ & $12 ; 7$ & $49 ; 3$ & $20 ; 2$ & - & - & - & 13 & - & - \\
\hline Xu et al., 2017 (40) & $30 ; 0$ & $4 ; 0$ & $15 ; 0$ & $2 ; 0$ & - & - & - & 4 & - & - \\
\hline
\end{tabular}

*, for studies reporting safety data on drug and placebo separately, data from both groups are provided; ${ }^{\dagger}$, AEs following the sixth cycle of treatment; ${ }^{\S}$ " treatment-emergent AEs. HTN, hypertension; HFSR, hand-foot skin reaction; C, placebo; I, intervention.

determines the safety and efficacy of a particular drug in the specified population $(53,54)$. Although several studies conducted in the Caucasian population have evaluated the efficacy and safety of regorafenib, there is no evidence on the same for fruquintinib. Moreover, limited evidence is available on the efficacy and safety of fruquintinib compared with regorafenib. Furthermore, as opposed to regorafenib, there is no real-world evidence to support the effectiveness of fruquintinib in clinical practice.

Participants enrolled in FRESCO comprised a lesser proportion of men in the fruquintinib group than in the placebo group, whereas the CONCUR trial had a higher proportion of women in the regorafenib arm compared with the placebo arm $(38,39)$. Epidemiological studies have demonstrated that the prevalence of CRC and related mortality rates are higher in men than in women $(3,4)$. As gender-related differences affect the pharmacokinetic and pharmacodynamic profile of a drug and hence influence treatment response $(55,56)$, a lower proportion of men in the fruquintinib arm might have positively affected the survival outcomes in the FRESCO trial. Furthermore, the CONCUR trial had a higher proportion of patients aged $\geq 65$ years and those who have received $>3$ lines of systemic therapy or metastatic disease treatment (expected to have poor prognosis) in the drug arm compared with that in the FRESCO trial $(38,39)$. To summarize, patients in the drug treatment arm in the CONCUR study had poor baseline characteristics in comparison with those in the fruquintinib arm in FRESCO, and these differences might have skewed the outcomes in FRESCO in favor of fruquintinib. These details should be taken into account while interpreting results of FRESCO and CONCUR, and while selecting the optimal third-line therapy for mCRC.

Although previous meta-analyses have compared the efficacy of regorafenib and fruquintinib, they had included only RCT evidence for statistical analysis. Although RCT evidence may provide good internal validity, in case of CRC, the patient age group in RCT settings cannot reflect 
real-world age distribution. Meta-analysis of real-world evidence has not been performed for comparing regorafenib and fruquintinib primarily because of the nonavailability of real-world evidence for assessing the effectiveness of fruquintinib. Moreover, the heterogeneity with respect to age in the different RCTs cannot be accounted for satisfactorily in meta-analysis. Further, while previous studies also included other drugs such as TAS-102, the current narrative review included only fruquintinib and regorafenib as both have similar modes of action. This is of significance considering that current studies are also evaluating the combination of angiogenic inhibitors and programmed cell death ligand-1inhibitors.

\section{Future perspectives}

With emerging anti-angiogenic agents, it seems that multi-targeted TKIs hold a therapeutic advantage over monoclonal antibodies because they can block multiple angiogenic signaling pathways simultaneously. Even though the mode of action of all anti-angiogenic receptor TKIs is the same, they differ in their pharmacokinetics and substance-specific AEs. Although international consensus over various therapy options has been made, an important question remains unresolved: how can antiangiogenic medicine be optimized. Future studies and trials should focus on exploring predictive biomarkers that will identify responders among the large patient group of nonresponders. To date, only a few candidates for predictive biomarkers have been identified but are from small studies (57).

A dose-escalation, multicenter non-RCT in the United States (US) evaluating the toxicity and anticancer activity of fruquintinib in patients with advanced solid tumors (NCT03251378) revealed that the anticancer activity, safety profile, and tolerability of fruquintinib were congruous with other anti-angiogenic TKIs. The recommended phase 2 dose of $5 \mathrm{mg}$ once daily in US patients is similar to that in Chinese patients; the dose-expansion phase of the study is still ongoing (58). FRESCO-2 (NCT04322539 and EudraCT: 2020-000158-88) is another key study evaluating the efficacy and safety of fruquintinib in patients with refractory mCRC. It is a randomized, phase 3 study being held at 136 locations, with recruitment in the US, Europe, Australia, and Japan. A total of 687 patients with progression on or intolerance to trifluridine-tipiracil and/ or regorafenib and previously treated with chemotherapy will be randomized to either fruquintinib or placebo in conjunction with best supportive care. This study will determine the OS and PFS and provide valuable insights into a diverse population (59).

Current understanding of the mechanisms of vascularization of micrometastatic lesions is very limited, and agents that can block other modes of tumor vascularization are needed. Furthermore, understanding the mechanistic differences between VEGFR TKIs and anti-VEGF antibodies will help in optimizing the design of anticancer treatments. With more and more anticancer agents available, it is a challenge for the oncologist to make an optimal choice in the sequence of treatment without evidence based on head-on trials. The results are awaited from a real-world study of regorafenib versus fruquintinib in CRC (NCT04431791), which will provide clarity on their clinical benefits. International guidelines have reached a global consensus for the choice of drugs in different lines of treatment, the optimal strategy in the third line, and the sequence of drugs for mCRC; however, this is yet to be resolved.

\section{Conclusions}

Current guidelines have approved regorafenib for thirdand later-line treatment of patients with mCRC. Owing to emerging targets, the best strategies for treating patients with mCRC will continue to evolve as long as there are new treatment combinations and novel therapies to improve outcomes for patients. Baseline characteristics of the population in the drug arm in the CONCUR trial were poor in comparison with those in the FRESCO trial, which negatively affected the outcomes. However, in terms of efficacy, both drugs offer similar outcomes. Further clinical studies investigating the current treatment options are underway as we continue to improve our understanding of the use of these therapies in real-world settings. Regorafenib and fruquintinib as third-line therapy options in CRC have offered promising outcomes in terms of safety and efficacy. However, further breakdown of safety characteristics suggests regorafenib as a clear choice for treatment. Nonetheless, future RCTs comparing the efficacy and safety of regorafenib and fruquintinib as thirdline therapy in mCRC are warranted to establish an optimal third-line treatment option for patients with mCRC.

\section{Acknowledgments}

Medical writing support under the authors' guidance was 
provided by Amit Bhat, PhD, CMPP (Indegene Pvt Ltd, Bangalore, India).

Funding: None.

\section{Footnote}

Reporting Checklist: The authors have completed the Narrative Review reporting checklist. Available at https:// tcr.amegroups.com/article/view/10.21037/tcr-20-3539/rc

Peer Review File: Available at https://tcr.amegroups.com/ article/view/10.21037/tcr-20-3539/prf

Conflicts of Interest: All authors have completed the ICMJE uniform disclosure form (available at https://tcr.amegroups. com/article/view/10.21037/tcr-20-3539/coif). The authors have no conflicts of interest to declare.

Ethical Statement: The authors are accountable for all aspects of the work in ensuring that questions related to the accuracy or integrity of any part of the work are appropriately investigated and resolved.

Open Access Statement: This is an Open Access article distributed in accordance with the Creative Commons Attribution-NonCommercial-NoDerivs 4.0 International License (CC BY-NC-ND 4.0), which permits the noncommercial replication and distribution of the article with the strict proviso that no changes or edits are made and the original work is properly cited (including links to both the formal publication through the relevant DOI and the license). See: https://creativecommons.org/licenses/by-nc-nd/4.0/.

\section{References}

1. World Health Organization. Colorectal cancer (Globocan 2020) International Agency for Research on Cancer. Accessed May 20, 2021. Available online: https://gco.iarc. fr/today/data/factsheets/cancers/10_8_9-Colorectum-factsheet.pdf

2. Arnold M, Sierra MS, Laversanne M, et al. Global patterns and trends in colorectal cancer incidence and mortality. Gut 2017;66:683-91.

3. White A, Ironmonger L, Steele RJC, et al. A review of sex-related differences in colorectal cancer incidence, screening uptake, routes to diagnosis, cancer stage and survival in the UK. BMC Cancer 2018;18:906.

4. Douaiher J, Ravipati A, Grams B, et al. Colorectal cancer- global burden, trends, and geographical variations. J Surg Oncol 2017;115:619-30.

5. Van Cutsem E, Nordlinger B, Cervantes A, et al. Advanced colorectal cancer: ESMO Clinical Practice Guidelines for treatment. Ann Oncol 2010;21 Suppl 5:v93-7.

6. Salvatore L, Aprile G, Arnoldi E, et al. Management of metastatic colorectal cancer patients: guidelines of the Italian Medical Oncology Association (AIOM). ESMO Open 2017;2:e000147.

7. Holch J, Stintzing S, Heinemann V. Treatment of Metastatic Colorectal Cancer: Standard of Care and Future Perspectives. Visc Med 2016;32:178-83.

8. Jing Z, Rui Z, Binglan Z. A comparison of regorafenib and fruquintinib for metastatic colorectal cancer: a systematic review and network meta-analysis. J Cancer Res Clin Oncol 2019;145:2313-23.

9. Qiu T, Chen W, Li P, et al. Subsequent anti-VEGF therapy after first-line anti-EGFR therapy improved overall survival of patients with metastatic colorectal cancer. Onco Targets Ther 2018;11:465-71.

10. Buchler T, Chloupkova R, Poprach A, et al. Sequential therapy with bevacizumab and EGFR inhibitors for metastatic colorectal carcinoma: a national registry-based analysis. Cancer Manag Res 2019;11:359-68.

11. Cicero G, De Luca R, Dieli F. Efficacy and Safety of the Oral Multikinase Regorafenib in Metastatic Colorectal Cancer. Oncology 2017;93:354-8.

12. Grothey A, Van Cutsem E, Sobrero A, et al. Regorafenib monotherapy for previously treated metastatic colorectal cancer (CORRECT): an international, multicentre, randomised, placebo-controlled, phase 3 trial. Lancet 2013;381:303-12.

13. Bayer. Stivarga $40 \mathrm{mg}$ film-coated tablets. Summary of product characteristics. 2017.

14. Geng F, Wang Z, Yin H, et al. Molecular Targeted Drugs and Treatment of Colorectal Cancer: Recent Progress and Future Perspectives. Cancer Biother Radiopharm 2017;32:149-60.

15. Shirley M. Fruquintinib: First Global Approval. Drugs 2018;78:1757-61.

16. Abou-Elkacem L, Arns S, Brix G, et al. Regorafenib inhibits growth, angiogenesis, and metastasis in a highly aggressive, orthotopic colon cancer model. Mol Cancer Ther 2013;12:1322-31.

17. Sun Q, Zhou J, Zhang Z, et al. Discovery of fruquintinib, a potent and highly selective small molecule inhibitor of VEGFR 1, 2, 3 tyrosine kinases for cancer therapy. Cancer Biol Ther 2014;15:1635-45. 
18. Takigawa H, Kitadai Y, Shinagawa K, et al. Multikinase inhibitor regorafenib inhibits the growth and metastasis of colon cancer with abundant stroma. Cancer Sci 2016;107:601-8.

19. Chen Z, Jiang L. The clinical application of fruquintinib on colorectal cancer. Expert Rev Clin Pharmacol 2019;12:713-21.

20. Majithia N, Grothey A. Regorafenib in the treatment of colorectal cancer. Expert Opin Pharmacother 2016;17:137-45.

21. Folkman J. Tumor angiogenesis: therapeutic implications. N Engl J Med 1971;285:1182-6.

22. Fallah A, Sadeghinia A, Kahroba H, et al. Therapeutic targeting of angiogenesis molecular pathways in angiogenesis-dependent diseases. Biomed Pharmacother 2019;110:775-85.

23. Lee WS, Kim J. 2018 Cardiotoxicity associated with tyrosine kinase-targeted anticancer therapy. Mol Cell Toxicol 2018;14:247-54.

24. Krasinskas AM. EGFR Signaling in Colorectal Carcinoma. Patholog Res Int 2011;2011:932932.

25. Hiret S, Borg C, Bertaut A, et al. Bevacizumab or cetuximab plus chemotherapy after progression with bevacizumab plus chemotherapy in patients with wtKRAS metastatic colorectal cancer: A randomized phase II study (Prodige 18 -UNICANCER GI). J Clin Oncol 2016;34:3514.

26. Li R, Liang M, Liang X, et al. Chemotherapeutic Effectiveness of Combining Cetuximab for Metastatic Colorectal Cancer Treatment: A System Review and MetaAnalysis. Front Oncol 2020;10:868.

27. Shitara K, Yamanaka T, Denda T, et al. REVERCE: a randomized phase II study of regorafenib followed by cetuximab versus the reverse sequence for previously treated metastatic colorectal cancer patients. Ann Oncol 2019;30:259-65.

28. Selvakumaran M, Yao KS, Feldman MD, et al. Antitumor effect of the angiogenesis inhibitor bevacizumab is dependent on susceptibility of tumors to hypoxia-induced apoptosis. Biochem Pharmacol 2008;75:627-38.

29. Rogosin S, Sandler AB. Beyond bevacizumab: antiangiogenic agents. Clin Lung Cancer 2012;13:326-33.

30. Fan F, Samuel S, Gaur P, et al. Chronic exposure of colorectal cancer cells to bevacizumab promotes compensatory pathways that mediate tumour cell migration. Br J Cancer 2011;104:1270-7.

31. Miyazaki S, Kikuchi H, Iino I, et al. Anti-VEGF antibody therapy induces tumor hypoxia and stanniocalcin 2 expression and potentiates growth of human colon cancer xenografts. Int J Cancer 2014;135:295-307.

32. Hayashi H, Arao T, Matsumoto K, et al. Biomarkers of reactive resistance and early disease progression during chemotherapy plus bevacizumab treatment for colorectal carcinoma. Oncotarget 2014;5:2588-95.

33. Cohen DJ, Hochster HS. Rationale for combining biotherapy in the treatment of advanced colon cancer. Gastrointest Cancer Res 2008;2:145-51.

34. Ding C, Li L, Yang T, et al. Combined application of anti-VEGF and anti-EGFR attenuates the growth and angiogenesis of colorectal cancer mainly through suppressing AKT and ERK signaling in mice model. BMC Cancer 2016;16:791.

35. Tonra JR, Deevi DS, Corcoran E, et al. Synergistic antitumor effects of combined epidermal growth factor receptor and vascular endothelial growth factor receptor-2 targeted therapy. Clin Cancer Res 2006;12:2197-207.

36. Saltz LB, Lenz HJ, Kindler HL, et al. Randomized phase II trial of cetuximab, bevacizumab, and irinotecan compared with cetuximab and bevacizumab alone in irinotecan-refractory colorectal cancer: the BOND-2 study. J Clin Oncol 2007;25:4557-61.

37. Briffa R, Langdon SP, Grech G, et al. Acquired and Intrinsic Resistance to Colorectal Cancer Treatment. Colorectal Cancer - Diagnosis, Screening and Management. IntechOpen. 2017. doi: 10.5772/ intechopen.70781

38. Li J, Qin S, Xu R, et al. Regorafenib plus best supportive care versus placebo plus best supportive care in Asian patients with previously treated metastatic colorectal cancer (CONCUR): a randomised, double-blind, placebocontrolled, phase 3 trial. Lancet Oncol 2015;16:619-29.

39. Li J, Qin S, Xu RH, et al. Effect of Fruquintinib vs Placebo on Overall Survival in Patients With Previously Treated Metastatic Colorectal Cancer: The FRESCO Randomized Clinical Trial. JAMA 2018;319:2486-96.

40. Xu RH, Li J, Bai Y, et al. Safety and efficacy of fruquintinib in patients with previously treated metastatic colorectal cancer: a phase Ib study and a randomized double-blind phase II study. J Hematol Oncol 2017;10:22.

41. Adenis A, de la Fouchardiere C, Paule B, et al. Survival, safety, and prognostic factors for outcome with Regorafenib in patients with metastatic colorectal cancer refractory to standard therapies: results from a multicenter study (REBECCA) nested within a compassionate use program. BMC Cancer 2016;16:412.

42. Kopeckova K, Buchler T, Bortlicek Z, et al. Regorafenib 
in the Real-Life Clinical Practice: Data from the Czech Registry. Target Oncol 2017;12:89-95.

43. Grothey A, Van Cutsem E, Sobrero AF, et al. 2013 Time course of regorafenib-associated adverse events in the phase III CORRECT study. J Clin Oncol 2013;31:467.

44. Komatsu Y, Muro K, Yamaguchi K, et al. Safety and efficacy of regorafenib post-marketing surveillance (PMS) in Japanese patients with metastatic colorectal cancer (mCRC). J Clin Oncol 2017;35:721.

45. Ducreux M, Petersen LN, Öhler L, et al. Safety and effectiveness of regorafenib in patients with metastatic colorectal cancer in routine clinical practice in the prospective, observational CORRELATE study. Eur J Cancer 2019;123:146-54.

46. Schulz H, Janssen J, Strauss UP, et al. Clinical efficacy and safety of regorafenib (REG) in the treatment of metastatic colorectal cancer (mCRC) in daily practice in Germany: Final results of the prospective multicentre noninterventional RECORA study. J Clin Oncol 2018;36:748.

47. Calcagno F, Lenoble S, Lakkis Z, et al. Efficacy, Safety and Cost of Regorafenib in Patients with Metastatic Colorectal Cancer in French Clinical Practice. Clin Med Insights Oncol 2016;10:59-66.

48. Van Cutsem E, Martinelli E, Cascinu S, et al. Regorafenib for Patients with Metastatic Colorectal Cancer Who Progressed After Standard Therapy: Results of the Large, Single-Arm, Open-Label Phase IIIb CONSIGN Study. Oncologist 2019;24:185-92.

49. Lam KO, Lee KC, Chiu J, et al. The real-world use of regorafenib for metastatic colorectal cancer: multicentre analysis of treatment pattern and outcomes in Hong Kong. Postgrad Med J 2017;93:395-400.

50. Cardone C, Martinelli E, Troiani T, et al. Exploratory findings from a prematurely closed international, multicentre, academic trial: RAVELLO, a phase III study of regorafenib versus placebo as maintenance therapy after first-line treatment in RAS wild-type metastatic colorectal cancer. ESMO Open 2019;4:e000519.

51. Yeh KH, Yang TS, Hsu TC, et al. Real-world evidence of the safety and effectiveness of regorafenib in Taiwanese patients with metastatic colorectal cancer: CORRELATE Taiwan. J Formos Med Assoc 2021;120:2023-31.

52. Kudo T, Kato T, Kagawa Y, et al. Phase II dose titration study of regorafenib for patients with unresectable metastatic colorectal cancer that progressed after standard chemotherapy. J Clin Oncol 2018;36:821.

53. Yasuda SU, Zhang L, Huang SM. The role of ethnicity in variability in response to drugs: focus on clinical pharmacology studies. Clin Pharmacol Ther 2008;84:417-23.

54. Ramamoorthy A, Pacanowski MA, Bull J, et al. Racial/ ethnic differences in drug disposition and response: review of recently approved drugs. Clin Pharmacol Ther 2015;97:263-73.

55. Kim HI, Lim H, Moon A. Sex Differences in Cancer: Epidemiology, Genetics and Therapy. Biomol Ther (Seoul) 2018;26:335-42.

56. Rubinow DR, Moore M. Sex-dependent modulation of treatment response. Dialogues Clin Neurosci 2004;6:39-51.

57. Jain RK, Duda DG, Willett CG, et al. Biomarkers of response and resistance to antiangiogenic therapy. Nat Rev Clin Oncol 2009;6:327-38.

58. Wang-Gillam A, Yeckes-Rodin H, Kosmo MA, et al. Phase I trial of fruquintinib in patients with advanced solid tumors: Results of the dose escalation phase. Ann Oncol 2019;30:v175-6.

59. Dasari A, Sobrero A, Yao J, et al. FRESCO-2: a global Phase III study investigating the efficacy and safety of fruquintinib in metastatic colorectal cancer. Future Oncol 2021;17:3151-62.
Cite this article as: $\mathrm{Xu} X, \mathrm{Yu} Y$, Liu M, Liang L, Liu T. Efficacy and safety of regorafenib and fruquintinib as thirdline treatment for colorectal cancer: a narrative review. Transl Cancer Res 2022;11(1):276-287. doi: 10.21037/tcr-20-3539 